Research Article

\title{
Analysis of the MHD Boundary Layer Flow over a Nonlinear Stretching Sheet in a Porous Medium Using Semianalytical Approaches
}

\author{
K. Jabeen (iD, M. Mushtaq, and R. M. Akram \\ Department of Mathematics, University of the Engineering and Technology Lahore, Lahore 54890, Pakistan \\ Correspondence should be addressed to K. Jabeen; kanwaljabeen44@yahoo.com
}

Received 1 November 2019; Revised 8 January 2020; Accepted 17 January 2020; Published 20 February 2020

Academic Editor: Giorgio Besagni

Copyright (c) $2020 \mathrm{~K}$. Jabeen et al. This is an open access article distributed under the Creative Commons Attribution License, which permits unrestricted use, distribution, and reproduction in any medium, provided the original work is properly cited.

\begin{abstract}
The purpose of the research is to inquire the outcomes of viscous and ohmic dissipation on the MHD flow in porous media in the region of suction and injection. A flow model of nonlinear ODEs with assisting boundary conditions is tackled with the help of computational software by using various standard techniques. The effects of relevant parameters on the concentration, thermal, and velocity distribution are illustrated graphically; also, the skin friction coefficient and flow rates of heat and mass transfer are calculated and shown in a tabular way. An analysis of the consequences proves that the flow field is effectively appreciable by injection and suction. Comparison with the already published work is made and found to be in good agreement.
\end{abstract}

\section{Introduction}

The boundary layer (BL) of velocity relates to the existence of shear stress and velocity gradient, the temperature BL is connected with the heat transfer and temperature gradient, while the concentration BL is associated with the concentration gradient and species transfer. The porous medium where the fluid is in motion may be responsible for the formation of these three BLs. These three layers are affected by each other. Hydromagnetics or magnetohydrodynamics is basically the study of the flow having magnetic characteristics and behavior of electrically transmitted fluids. The basic idea regarding the MHD $[1,2]$ is that, in a moving conductive fluid, the magnetic field induces current which enhances the fluid to get polarized and changes the magnetic field itself reciprocally. Some common examples of magnetofluids are electrolytes, salt water, liquid metals, and plasma. The initiative in the field of MHD was firstly taken by Faraday in 1832 in which he proposed the concept about the interaction of the sea flow with earth as a magnetic field. Later, in 1937, Lazarus and Hartman found the exact solutions of MHD equations for the first time in the history. They had also performed some experiments on the liquid metal flow in the MHD channel. After them, in 1942, Hannes Alfven described the class of MHD waves, later known as Alfven waves. For the achievements in the field of MHD, he was conferred an honorary Nobel prize in 1970. Currently, MHD has a wide range of applications in geophysics, astrophysics, and agriculture sciences $[3,4]$. Also in the technical field, the MHD study has considerable interest because of its fruitful applications in the industrial field, for instance, MHD liquid metal flow control, cooling of nuclear reactors, high-temperature plasma, MHD power generators, and biological transportation. Since the last few decades, the study of a 2D boundary layer flow (BLF) of incompressible and viscous fluids around a shrinking/stretching sheet is worthy of attention due to its vast applications in engineering disciplines [2-4]. Sakiadis was the pioneer who had firstly explored the flow around a stretching sheet, while Erickson et al. augmented his work to include suction at a stretching sheet and examined the results on mass and heat transfer on the BL. Later, the work had been escalated for different physical situations by many researchers. Ferdows et al. had examined the influence of large suction on the MHD flow by a porous vertical plate. Lin et al. considered the presence of the electrically conducting second-grade 
fluid flow of the transverse magnetic field over a stretching sheet. Over this sheet, Gupta [5] had examined the mass and heat transfer. Continuing Crane's work, Chem et al. and Dutta et al. under the different physical phenomena considered the influence of mass and heat transfer analysis. The effects of constant viscosity on the fluid characteristics were investigated by Mehta and Gray et al. Around a stretching sheet, the Dufour and Soret effects in the magnetic field were examined by Ahammad and Mollah [3, 6] numerically. Bhargava and Chandra had studied the numerical solution of heat transfer analysis and MHD flow around a nonlinear stretching sheet fixed in a porous medium [7]. Also, Alinejad and Samarbakhsh [4] investigated viscous flow with heat dissipation on a nonlinear stretching sheet $[1,8]$, while Zaimi et al. worked with a permeable stretching sheet with viscous effects. Later, Dessie et al. [8, 9, 10] amplified Zaimi's work by making a part of MHD flow with viscous dissipation effects [7]. Recently, Oderinu et al. and Bhargava et al. $[7,8]$ solved the MHD partial differential equations and obtained the results in the form of series solutions with slip BCs in the porous medium. In many extents of engineering and industrial areas of interest, the flow through porous media plays an important role in MHD flow meters, MHD pumps, and MHD power generators $[3,4]$. Moreover, in polymer industries, wide range of applications are to be found in a particular flow on a stretching sheet. A little while back, Anwar et al. and Cortell et al. have studied viscous conducting fluid flows around a stretching sheet with the constant rate of stretching [6, 11]. Sharma et al. [12] had inquired the influence of chemical reaction and ohmic dissipation in the permeable medium, and Pattnaik et al. [13] had studied the MHD flow with constant suction/injection in a porous medium. Prakash et al. [14] worked on the influence of the MHD nanofluid flow on the inclined stretching sheet under viscous and ohmic heating effects. Recently, viscous and ohmic dissipation on magnetohydrodynamic nanofluid flow in the porous medium had been analyzed with suction/injection effects by Jagadha et al. [14, 15]. Also, various semianalytical approaches for the MHD squeezing flow had been thoroughly discussed by Ullah et al. [16], while Jabeen et al. [17] had presented a comparison of semianalytical methods for the MHD flow in the porous medium. Mabood et al. [17, 18] numerically analyzed the multiple slip results on the MHD unsteady flow of mass and heat transfer around a stretching sheet. Emeka [19] reported the viscous dissipation and Soret influence on the MHD fluid flow with thermal radiation. A basic objective of the current study is to inquire the mass and heat transfer rates on a steady and viscous fluid flow around a nonlinear stretching sheet fixed in a permeable medium under the influence of viscous and ohmic dissipation, Eckert number, Soret number, and Prandtl number. The set of MHD equations is actually the amalgamation of Navier-Stokes and Maxwell equations. For this purpose, to overcome the difficulties concerning the closed-form solution of the nonlinear boundary value problem, some semianalytical methods are taken into account. Many other research studies in the literature with great interest have largely used perturbation techniques HPM, HAM, ADM, and successive approximation method. But in this work, we will mainly focus on the behavior of ADM, DTM, and VIM along with Pade approximation $[6,17,18]$. For the solution of nonlinear and linear ODEs and PDEs, these methods are strongly effective and reliable which can be applied directly to nonlinear equations in physics, engineering, and mathematics. Although these methods have some drawbacks, they are applicable in the small region although invalid in an unbound domain. To tackle this issue, we have considered Pade approximation $[17,19]$.

\section{Mathematical Formulation}

We consider the steady $2 \mathrm{D}$ viscous incompressible flow of the electrically transmitted fluid around a nonlinear stretching sheet fixed in a porous medium. The applied magnetic field acts in the perpendicular direction to the flow with uniform strength $B_{0}$. The magnetic field is assumed insignificant as the magnetic Reynolds number is considered small enough. The physical configuration of the flow regime is presented in Figure 1.

The basic two-dimensional BL equations subject to the above consideration are

$$
\begin{aligned}
& \frac{\partial u}{\partial x}+\frac{\partial v}{\partial y}=0 \\
& u \frac{\partial u}{\partial x}+v \frac{\partial u}{\partial y}=\widehat{U} \frac{\mathrm{d} \widehat{U}}{\mathrm{~d} x}+v \frac{\partial^{2} u}{\partial y^{2}}-\sigma \frac{B^{2}(x)(u-\widehat{U})}{\rho}-\frac{v(u-\widehat{U})}{k_{p^{\prime}}} \\
& u \frac{\partial T}{\partial x}+v \frac{\partial T}{\partial y}=\frac{k}{\rho C_{p}} \frac{\partial^{2} T}{\partial y^{2}}+\frac{\mu}{\rho C_{p}}\left(\frac{\partial u}{\partial y}\right)^{2}-\frac{1}{\rho C_{p}} \frac{\partial q_{r}}{\partial y}+\frac{\sigma B^{2} u^{2}}{\rho C_{p}} \\
& u \frac{\partial C}{\partial x}+v \frac{\partial C}{\partial y}=D \frac{\partial^{2} C}{\partial y^{2}}+D_{m} \frac{k_{T}}{T_{m}} \frac{\partial^{2} T}{\partial y^{2}},
\end{aligned}
$$

where $u$ and $v$ are the velocity components, $v$ is the coefficient of kinematic viscosity, $\rho$ is the fluid density, $B$ is the strength of the induced magnetic field, $T$ is the temperature, $k /\left(\rho C_{p}\right)$ is the thermal diffusivity, $q_{r}$ is the radiative heat flux, $C$ is the concentration of the fluid, $D_{m}$ are the mass diffusion coefficients, and $T_{m}$ and $k_{T}$ are the mean temperature and the ratio of thermal diffusion. Also, $U_{w}=c x^{n}(n>1)$ is the stretching velocity of the sheet, and $B(x)=B_{0}(x) x^{(n-1) / 2}$ and $k_{p}(x)=k_{p^{\prime}} x^{1-n}$ are the variable magnetic field and variable permeability, respectively.

Governing equations associated with the BCs are as follows:

$$
\left\{\begin{array}{l}
y=0: u(x, 0)=0, v(x, 0)=v_{w}, T=T_{w}=T_{\infty}+T_{0} x^{2 n} \\
C=C_{w}=C_{\infty}+C_{0} x^{2 n} \\
y \longrightarrow \infty: u(x, y) \longrightarrow U_{w}(x), T \longrightarrow T_{\infty}, C \longrightarrow C_{\infty}
\end{array}\right.
$$

where $v_{w}=v_{0} x^{(n-1) / 2}$ is the blowing/suction velocity. For the case of suction, $v_{w}<0$, and for the case of blowing, $v_{w}>0$. 


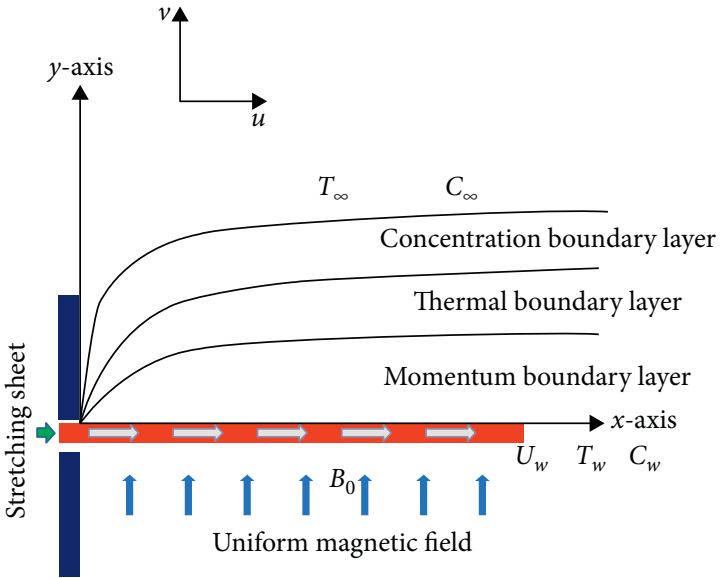

FIgURe 1: Physical interpretation of the flow model.

Also, $T_{0}$ and $C_{0}$ are positive concentration and temperature references. The radiative heat flux term in the temperature equation is simplified by the Rosseland approximation, i.e., $q_{r}=-\left(4 \sigma^{*} / 3 k^{*}\right)\left(\partial T^{4} / \partial y\right)$, where $\sigma^{*}$ is the Stefan-Boltzmann constant and $k^{*}$ is the mean absorption coefficient. Temperature variation in the flow is supposed to be small enough, so $T^{4}$ can be considered a linear function of temperature; neglecting the higher order term while expanding $T^{4}$ in Taylòr series about $T_{\infty}$ is obtained as $T^{4} \cong$ $4 T T_{\infty}^{3}-3 T_{\infty}^{3}$ as taken by the authors in $[12,14,19]$. The continuity equation is satisfied by choosing the stream function $\Psi$ which is

$$
\begin{gathered}
u=\frac{\partial \Psi}{\partial y}, \\
v=-\frac{\partial \Psi}{\partial x} .
\end{gathered}
$$

Introducing similarity transformation $[2,13,20]$,

$$
\begin{aligned}
\eta(x, y) & =x^{(n-1) / 2} y \sqrt{\frac{c(n+1)}{2 \nu}}, \\
\Psi(x, y) & =f(\eta) \sqrt{\frac{2 x \hat{U} v}{(n+1)}} \\
u & =c x^{n} f^{\prime}(\eta), \\
v & =-\sqrt{\frac{c \nu(n+1)}{2}} x^{(n-1) / 2}\left[f+\left(\frac{n-1}{n+1}\right) \eta f^{\prime}(\eta)\right] .
\end{aligned}
$$

$\theta(\eta)=\left(\left(T-T_{\infty}\right) /\left(T_{w}-T_{\infty}\right)\right)$ and $\phi(\eta)=\left(\left(C-C_{\infty}\right) /\right.$ $\left.\left(C_{w}-C_{\infty}\right)\right)$. The system of transformed nonlinear ODEs is

$$
f^{\prime \prime \prime}-\frac{2 n}{n+1}\left(f^{\prime 2}-1\right)+f f^{\prime \prime}-\left(M+\frac{1}{k_{p}}\right)\left(f^{\prime}-1\right)=0,
$$

$$
\begin{gathered}
\theta^{\prime \prime}+\frac{\operatorname{Pr}}{R+1} \theta^{\prime} f-2\left(\frac{\operatorname{Pr}}{R+1}\right)\left(\frac{2 n}{n+1}\right) \theta f^{\prime} \\
+\operatorname{Ec} \frac{\operatorname{Pr}}{R+1}\left(f^{\prime \prime 2}+M f^{\prime 2}\right)=0, \\
\phi^{\prime \prime}-\operatorname{Sc}\left(2\left(\frac{2 n}{n+1}\right) \phi f^{\prime}-f \phi^{\prime}\right)+\operatorname{ScS} \theta_{0} \theta^{\prime \prime}=0 .
\end{gathered}
$$

Also, the transformed BCs are

$$
\left\{\begin{array}{l}
f(0)=s ; f^{\prime}(0)=0 \\
f^{\prime}(\infty)=1 ; \theta(0)=1 ; \theta(\infty)=0 ; \phi(0)=1 ; \phi(\infty)=0
\end{array}\right.
$$

where dimensionless parameters in (5)-(7) such as magnetic field, permeability parameters, Prandtl, Eckert, thermal radiation, Schmidt, and Soret numbers are, respectively:

$$
\begin{aligned}
M & =\frac{2 \sigma B_{0}^{2}(x)}{c(n+1) \rho}, \\
K p & =\frac{2 \nu}{c(n+1) k p^{\prime \prime}}, \\
\operatorname{Pr} & =\frac{\mu C_{p}}{k}, \\
\mathrm{Ec} & =\frac{c^{2}}{T_{0} C_{p}}, \\
R & =\frac{16 \sigma^{*} T_{\infty}^{3}}{3 k^{*} k}, \\
\mathrm{Sc} & =\frac{\nu}{D^{\prime}} \\
\mathrm{Sr} & =\frac{D_{m} T_{0} k_{T}}{\nu C_{0} T_{m}} .
\end{aligned}
$$

\section{Results and Discussion}

The set of nonlinear ODEs (2)-(6) with described BCs is solved with the symbolic computational software MAPLE by using various standard techniques. The comparison with previous studies is discussed in Tables 1 and $2[2,8]$. The skin friction coefficient $f^{\prime \prime}(0)$, the rate of heat transfer $\theta^{\prime}(0)$, and the mass transfer rate $\phi^{\prime}(0)$ are tabulated in Tables 3-5 for various values of relevant parameters. Figures 2(a), 2(e), and $2(\mathrm{k})$ depict the behavior of the magnetic field on the velocity profile. It is described in an electrically conducting fluid that the magnetic field presence produces Lorentz force which acts reversely in the direction of the flow if the magnetic field is enforced in the perpendicular direction which slows down the fluid velocity. Figures 2(b), 2(f), and 2(l) describe the behavior of the permeability parameter $k p$. It is observed that as we increase the value of $k p$, the velocity profile increases. Physically, whenever in the porous medium, the holes are large enough and then the resistance of the porosity medium 
TABle 1: Comparison of $-f^{\prime \prime}(0)$ for $n=2$ and $k p=0$.

\begin{tabular}{lccccr}
\hline$M$ & $s$ & $\mathrm{ADM}_{[8,8]},-f^{\prime \prime}(0)$ & $\mathrm{DTM}_{[8,8]},-f^{\prime \prime}(0)$ & $\mathrm{VIM}_{[8,8]},-f^{\prime \prime}(0)$ & Su and Oderinu $[2,8],-f^{\prime \prime}(0)$ \\
\hline $3^{2}$ & 1 & 3.853408997 & 3.853408978 & 3.853408820 & 3.858873112 \\
$3^{2}$ & $-1 / 5$ & 3.208456549 & 3.208456544 & 3.208456457 & 3.207744568 \\
$5^{2}$ & 1 & 5.720272477 & 5.720272471 & 5.720272393 & 5.720170862 \\
$5^{2}$ & $-1 / 5$ & 5.090505123 & 5.090505119 & 5.090505109 & 5.090866724 \\
$10^{2}$ & 1 & 10.61013028 & 10.61013011 & 10.61013000 & 10.610546130 \\
$10^{2}$ & $-1 / 5$ & 9.996771590 & 9.996771582 & 9.996771561 & 9.996989268 \\
$15^{2}$ & 1 & 15.57313893 & 15.57313867 & 15.57313860 & 15.573606750 \\
$15^{2}$ & $-1 / 5$ & 14.96458586 & 14.96458521 & 14.96458517 & 14.964886980 \\
$20^{2}$ & 1 & 20.55459311 & 20.55459300 & 20.55459278 & 20.555144660 \\
$20^{2}$ & $-1 / 5$ & 19.94833712 & 19.94833678 & 19.94833612 & 19.948732630 \\
\hline
\end{tabular}

TABle 2: Comparison of $-f^{\prime \prime}(0)$ for $n=-3 / 5$ and $k p=0$.

\begin{tabular}{lccccc}
\hline$M$ & $s$ & $\operatorname{ADM}_{[8,8]},-f^{\prime \prime}(0)$ & $\mathrm{DTM}_{[8,8]},-f^{\prime \prime}(0)$ & $\mathrm{VIM}_{[8,8]},-f^{\prime \prime}(0)$ & Su and Oderinu $[2,8]$ \\
\hline $3^{2}$ & 1 & 2.813600000 & 2.813600000 & 2.813600000 & 2.813600000 \\
$3^{2}$ & $-1 / 5$ & 2.182752549 & 2.182752539 & 2.182752533 & 2.180130322 \\
$5^{2}$ & 1 & 5.1249775695 & 5.1249775691 & 5.1249775687 & 5.123657682 \\
$5^{2}$ & $-1 / 5$ & 4.503136617 & 4.503136610 & 4.503136602 & 4.502770284 \\
$10^{2}$ & 1 & 10.31627747 & 10.31627742 & 10.31627737 & 10.318228320 \\
$10^{2}$ & $-1 / 5$ & 9.705787139 & 9.705787131 & 9.705787121 & 9.707174832 \\
$15^{2}$ & 1 & 15.37904023 & 15.37904010 & 15.37904021 & 15.379648050 \\
$15^{2}$ & $-1 / 5$ & 14.77170266 & 14.77170263 & 14.77170251 & 14.772098060 \\
$20^{2}$ & 1 & 20.40938039 & 20.40938039 & 20.40938027 & 19.409974060 \\
$20^{2}$ & $-1 / 5$ & 19.80381019 & 19.80381014 & 19.80381011 & 19.804236230 \\
\hline
\end{tabular}

TABLE 3: Analysis of the skin friction coefficient.

\begin{tabular}{lccccc}
\hline$k p$ & $M$ & $s$ & $\mathrm{ADM},-f^{\prime \prime}(0)$ & $\mathrm{DTM},-f^{\prime \prime}(0)$ & $\mathrm{VIM},-f^{\prime \prime}(0)$ \\
\hline & 1 & & 1.891160019 & 1.891160019 & 1.891160019 \\
& 2 & & 2.139637171 & 2.139637171 & 2.139637169 \\
0.5 & 3 & 0.2 & 2.364507719 & 2.364507720 & 2.364507717 \\
& 4 & & 2.571112401 & 2.571112404 & 2.571112400 \\
& 5 & & 2.763170037 & 2.763170039 & 2.763170036 \\
0.5 & & & 2.139637171 & 2.139637171 & 2.139637169 \\
1 & & & 1.891160019 & 1.891160019 & 1.891160018 \\
5 & 2 & 0.2 & 1.670650146 & 1.670650147 & 1.670650144 \\
10 & & & 1.641547225 & 1.641547225 & 1.641547223 \\
100 & & & 1.615061966 & 1.615061967 & 1.615061966 \\
& & -2 & 4.083803044 & 4.083803039 & 4.083803043 \\
& & -1 & 3.022840970 & 3.022840970 & 3.022840969 \\
0.5 & 2 & 0 & 2.262565822 & 2.262565822 & 2.262565822 \\
& & 1 & 1.726627781 & 1.726627780 & 1.726627779 \\
& & 2 & 1.351825897 & 1.351825896 & 1.351825897 \\
\hline
\end{tabular}

can be neglected. So the velocity profile increases because the presence of the permeable surface increases the resistance to the fluid. In Figures 2(c), 2(j), and 2(o), suction and injection parameters are obtained on velocity, thermal, and concentration profiles. It is observed that, on a stretching sheet, suction on the BL slows down the backflow, whereas injection increases the reverse flow strength. It is observed in Figure $2(\mathrm{~g})$ that thermal $\mathrm{BL}$ thickness reduces when the Prandtl number $(\mathrm{Pr})$ increases. Pr is the ratio of momentum diffusivity to thermal diffusion. So when $\operatorname{Pr}$ gets smaller, the thermal BL thickness becomes larger as compared to the velocity $\mathrm{BL}$ thickness. Also, thermal BL thickness reduces when $\mathrm{Pr}$ increases. Figure 2(d) shows the variation in the velocity profile. It is clearly seen that when we increase the value of $n$, the velocity profile decreases. Figure $2(\mathrm{~h})$ shows the heat transfer dissipation factor or Eckert number (Ec) indicating the interrelationship between the fluid flow's kinetic energy and enthalpy differences of the BL. It expresses the conversion of kinetic energy into internal energy. So, it is observed that the thermal BL thickness increases when viscous dissipation increases.

In Figure 2(i), the effects of the thermal radiation parameter $R$ have been observed. It is noticed in the graphical representation that, with the increment of the value of $R$, the thermal BL thickness increases which is due to the fact that the radiation in the thermal BL increases when the value of $R$ increases. So the temperature gets higher, which causes the increment of the temperature profile.

In Figure 2(m), the Schmidt number (Sc) effects are presented graphically, and it is noticed that as we increase the value of Sc, the concentration profile decreases which indicates the physical phenomenon that the increment of Sc means the reduction of molecular diffusion Dm. The thermomigration or Soret number $(\mathrm{Sr})$ effects are graphically shown in Figure 2(n). Sr is basically the ratio of temperature difference towards the concentration, which depicts the mass transfer rate from lower solute concentration to higher concentration. Figure 2(n) illustrates that, 
TABLE 4: Numerical analysis for the rate of heat transfer.

\begin{tabular}{|c|c|c|c|c|c|c|c|c|}
\hline$M$ & $\operatorname{Pr}$ & $R$ & Ec & $s$ & $k p$ & $\mathrm{ADM},-\theta^{\prime}(0)$ & DTM, $-\theta^{\prime}(0)$ & VIM, $-\theta^{\prime}(0)$ \\
\hline 1 & 0.2 & 2 & 0.2 & 0.2 & 0.5 & 0.2004219216 & 0.2004219216 & 0.2004219218 \\
\hline 2 & & & & & & 0.1862471490 & 0.1862471488 & 0.1862471491 \\
\hline 3 & & & & & & 0.1768630818 & 0.1768630817 & 0.1768630820 \\
\hline 4 & & & & & & 0.1695720722 & 0.1695720721 & 0.1695720724 \\
\hline \multirow[t]{26}{*}{5} & & & & & & 0.1633084123 & 0.1633084120 & 0.1633084126 \\
\hline & 1 & & & & & 0.5181874225 & 0.5181874226 & 0.5181874226 \\
\hline & 2 & & & & & 0.8377493221 & 0.8377493215 & 0.8377493220 \\
\hline & 3 & & & & & 1.118794602 & 1.118794603 & 1.118794603 \\
\hline & 4 & & & & & 1.375513947 & 1.375513946 & 1.375513947 \\
\hline & 5 & & & & & 1.613375966 & 1.613375966 & 1.613375964 \\
\hline & & 1 & & & & 0.2382065096 & 0.2382065097 & 0.2382065099 \\
\hline & & 2 & & & & 0.1862471490 & 0.1862471488 & 0.1862471491 \\
\hline & & 3 & & & & 0.1570565842 & 0.1570565842 & 0.1570565843 \\
\hline & & 4 & & & & 0.1378980821 & 0.1378980820 & 0.1378980821 \\
\hline & & 5 & & & & 0.1241511891 & 0.1241511891 & 0.1241511889 \\
\hline & & & 1 & & & 0.1776365221 & 0.1776365220 & 0.1776365221 \\
\hline & & & 2 & & & 0.1862471490 & 0.1862471488 & 0.1862471489 \\
\hline & & & 3 & & & 0.1973123271 & 0.1973123271 & 0.1973123276 \\
\hline & & & 4 & & & 0.2091364830 & 0.2091364830 & 0.2091364836 \\
\hline & & & 5 & & & 0.2212901517 & 0.2212901516 & 0.2212901531 \\
\hline & & & & -2 & & 0.2620663019 & 0.2620663013 & 0.2620663021 \\
\hline & & & & -1 & & 0.2142537481 & 0.2142537478 & 0.2142537483 \\
\hline & & & & 0 & & 0.1892526045 & 0.1892526045 & 0.1892526044 \\
\hline & & & & 1 & & 0.1790179589 & 0.1790179588 & 0.1790179589 \\
\hline & & & & 2 & & 0.1774686453 & 0.1774686449 & 0.1774686451 \\
\hline & & & & & 0.5 & 0.1862471490 & 0.1862471488 & 0.1862471491 \\
\hline & & & & & 1 & 0.1769060879 & 0.1769060879 & 0.1769060881 \\
\hline & & & & & 5 & 0.1694451141 & 0.1694451141 & 0.1694451141 \\
\hline & & & & & 10 & 0.1685375984 & 0.1685375984 & 0.1685375984 \\
\hline & & & & & 100 & 0.1677308237 & 0.1677308237 & 0.1677308239 \\
\hline
\end{tabular}

TABle 5: Numerical analysis for the rate of mass transfer.

\begin{tabular}{|c|c|c|c|c|c|c|c|}
\hline$M$ & $\mathrm{Sc}$ & So & $k p$ & $s$ & $\mathrm{ADM},-\phi^{\prime}(0)$ & DTM, $-\phi^{\prime}(0)$ & VIM, $-\phi^{\prime}(0)$ \\
\hline 1 & 0.2 & 0.2 & 0.5 & 0.2 & 0.3426825062 & 0.3426825061 & 0.3426825060 \\
\hline 2 & & & & & 0.3384935640 & 0.3384935640 & 0.3384935642 \\
\hline 3 & & & & & 0.3369588494 & 0.3369588492 & 0.3369588490 \\
\hline 4 & & & & & 0.3367642385 & 0.3367642384 & 0.3367642385 \\
\hline \multirow[t]{3}{*}{5} & & & & & 0.3343770731 & 0.3343770730 & 0.3343770729 \\
\hline & 1 & & & & 0.7179016018 & 0.7183601774 & 0.7180726961 \\
\hline & 2 & & & & 0.9336448954 & 0.933875437 & 0.9336050934 \\
\hline \multirow[t]{17}{*}{2} & 3 & & & & 1.0556000222 & 1.0565534016 & 1.0506248616 \\
\hline & 4 & & & & 1.1472259621 & 1.1460100182 & 1.1460248613 \\
\hline & 5 & & & & 1.2218952183 & 1.2203615691 & 1.2235583812 \\
\hline & & 0.5 & & & 0.3427849385 & 0.3432842417 & 0.345832374 \\
\hline & & 1 & & & 0.3618297300 & 0.3623399123 & 0.3606979511 \\
\hline & & 2 & & & 0.3817816708 & 0.3801957518 & 0.3812702917 \\
\hline & & 3 & & & 0.4493500866 & 0.4489353684 & 0.449178977 \\
\hline & & & 0.5 & & 0.33849356401 & 0.33849356400 & 0.33849356404 \\
\hline & & & 1 & & 0.3445912661 & 0.3445672185 & 0.34456725657 \\
\hline & & & 5 & & 0.3542955163 & 0.3542955162 & 0.3542955167 \\
\hline & & & 10 & & 0.3561227939 & 0.3561227936 & 0.3561227940 \\
\hline & & & 100 & & 0.3579512214 & 0.3579512212 & 0.3579512210 \\
\hline & & & & -2 & 0.38383010775 & 0.38383010776 & 0.38383010771 \\
\hline & & & & -1 & 0.3529765977 & 0.3529765974 & 0.3529765972 \\
\hline & & & & 0 & 0.3421211429 & 0.3421211431 & 0.3421211431 \\
\hline & & & & 1 & 0.3243911722 & 0.3243911720 & 0.3243911719 \\
\hline & & & & 2 & 0.3170037444 & 0.3170037468 & 0.3170037446 \\
\hline
\end{tabular}




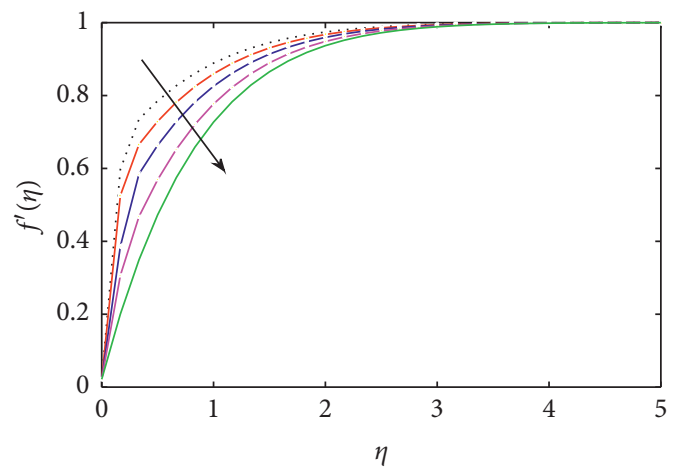

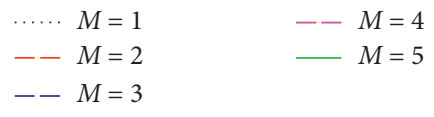

(a)
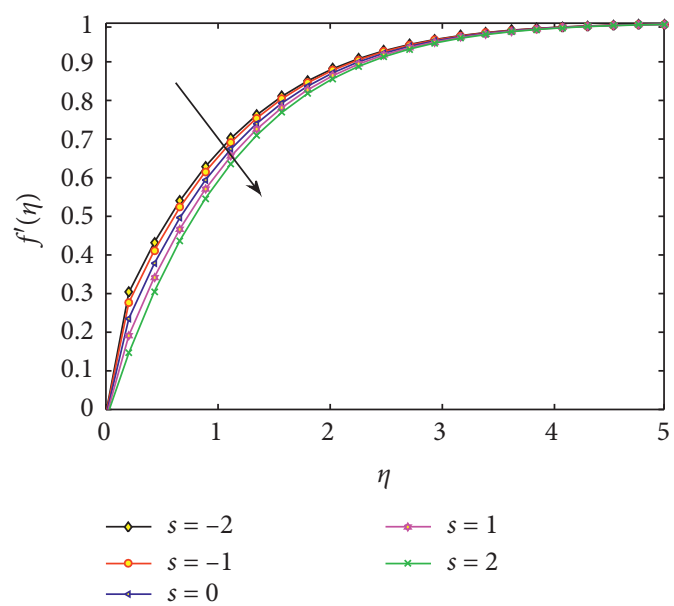

(c)

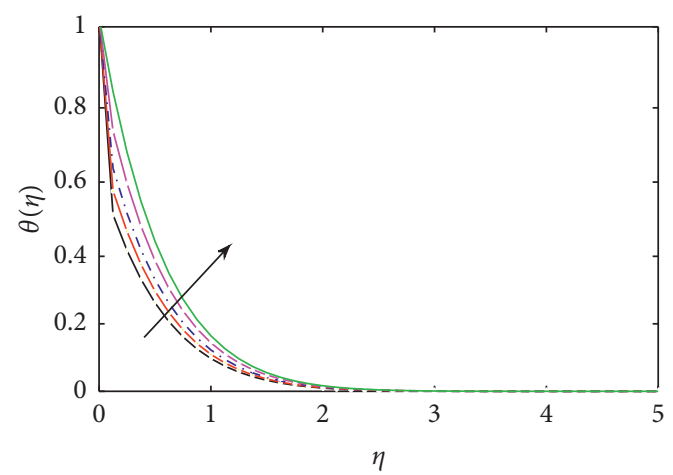

$-M=1$

$--M=2$

$-\longrightarrow M=4$

$M=5$

...- $M=3$
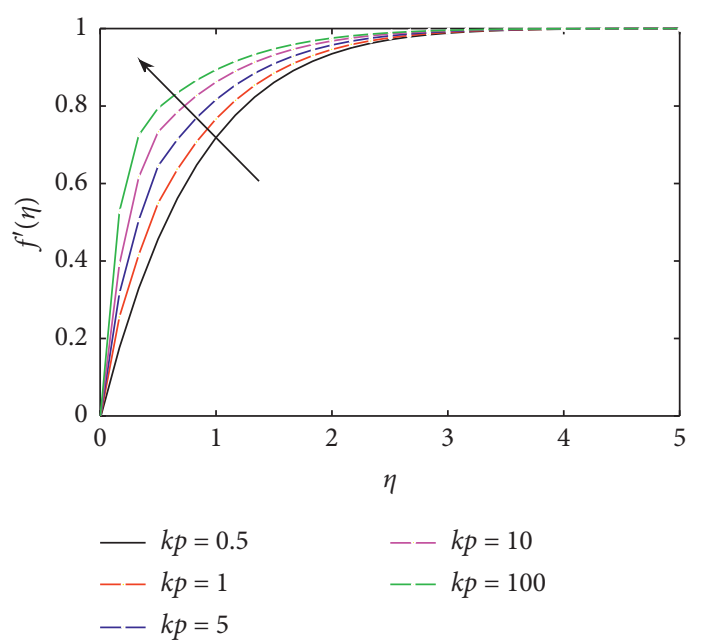

(b)
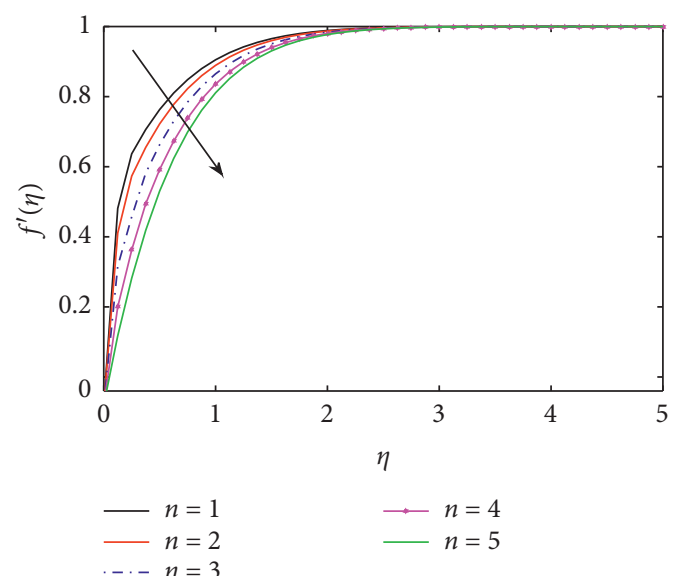

(d)

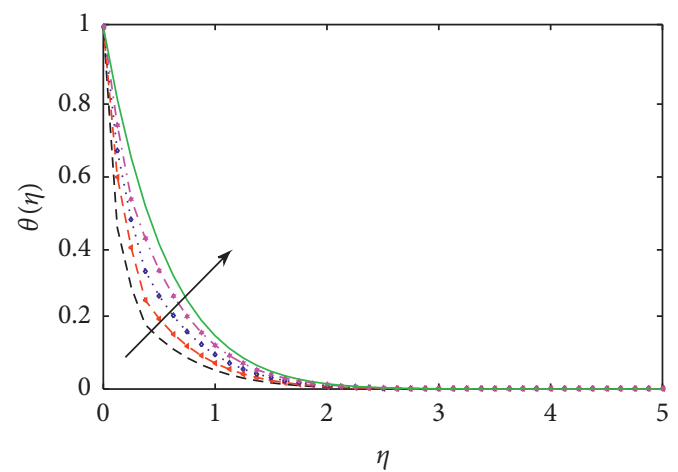
- - $k p=0.5$
$-k p=10$
$-* k p=1$
$-k p=100$
…. $k p=5$

(f)

Figure 2: Continued. 

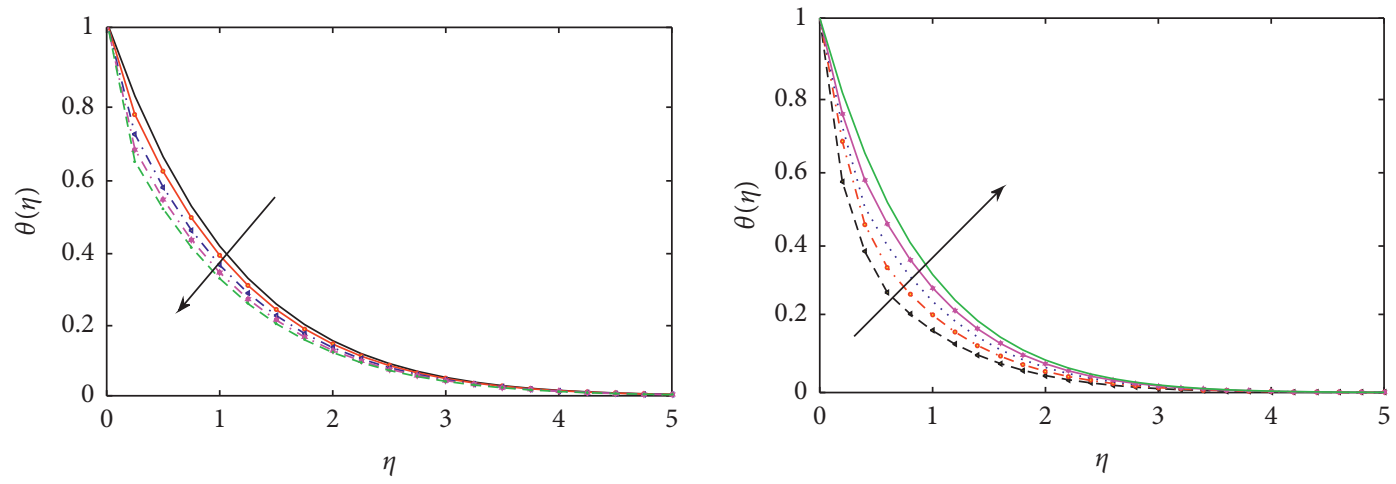

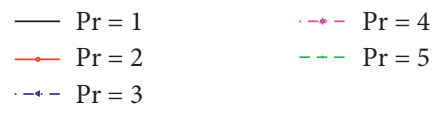

(g)

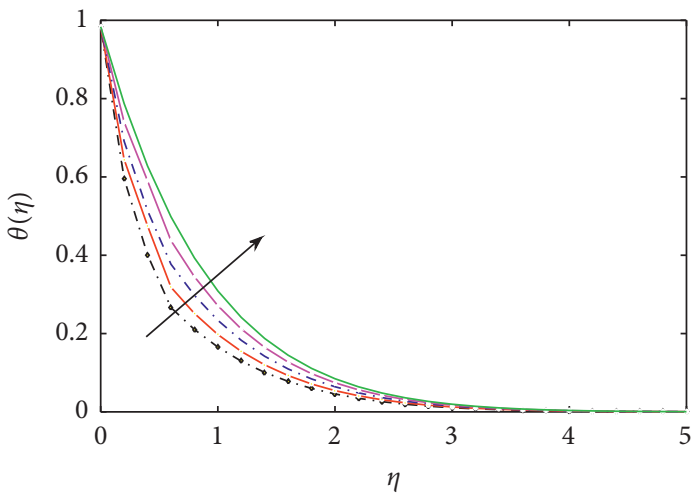

$$
\rightarrow-R=1
$$

$--R=3$

-. - $R=5$

(i)

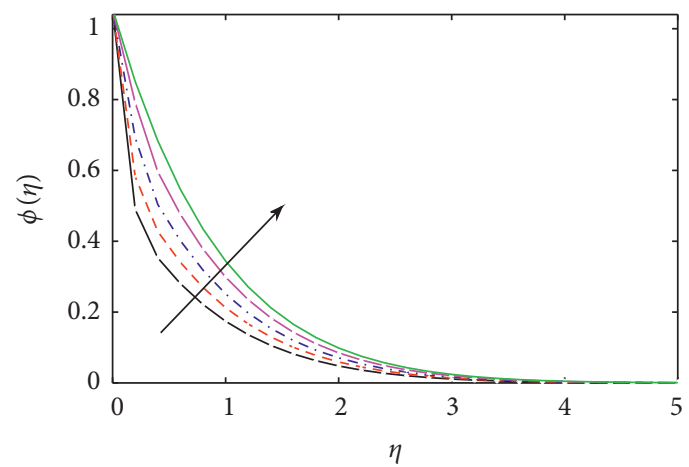

$$
\begin{aligned}
--\mathrm{Ec} & =1 \\
-\mathrm{Ec} & =2
\end{aligned}
$$$$
\text { …. } \mathrm{Ec}=3
$$

(h)
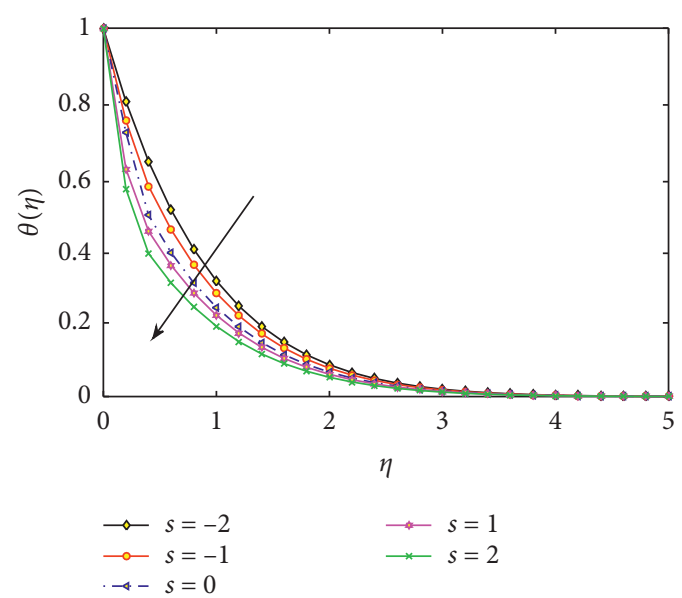

(j)

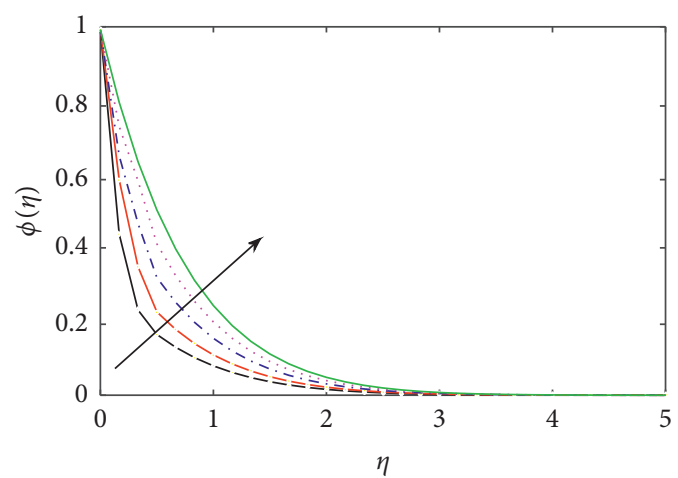

$$
\begin{aligned}
& -k p=0.5 \\
& --k p=1 \\
& -\cdots k p=5
\end{aligned}
$$

… $k p=10$

— $k p=100$

(l)

FIgURE 2: Continued. 


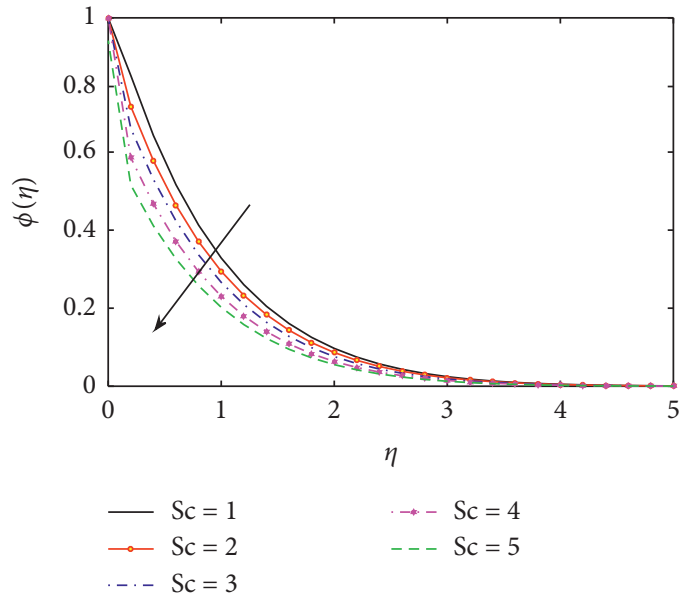

$(\mathrm{m})$

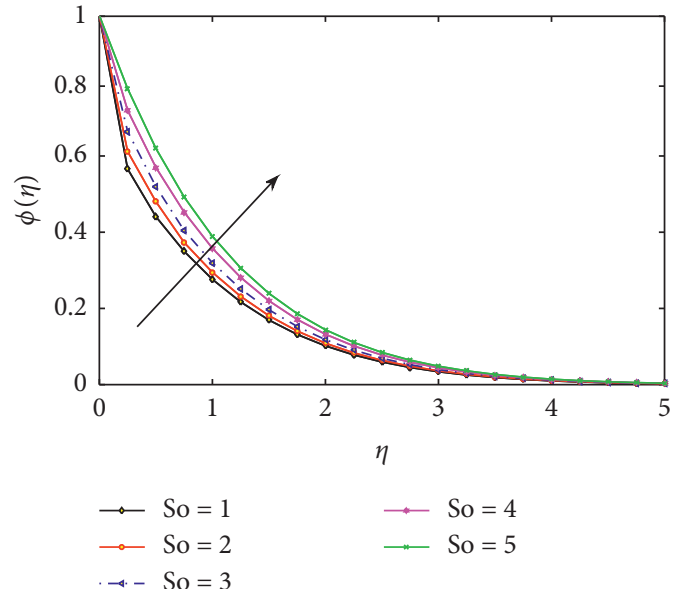

(n)

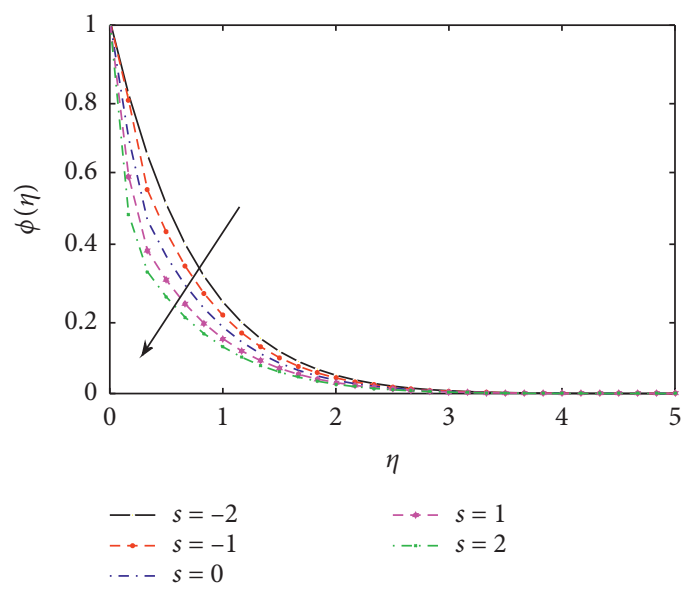

(o)

Figure 2: (a) $k p=0.5, \quad \operatorname{Pr}=\mathrm{Ec}=\mathrm{Sc}=\mathrm{So}=n=s=0.2, \quad R=7 ; \quad$ (b) $\quad M=2, \quad \operatorname{Pr}=E c=S c=S o=n=s=0.2, \quad R=7 ; \quad(\mathrm{c}) \quad k p=0.5, \quad \mathrm{M}=2$, $\mathrm{Ec}=\operatorname{Pr}=n=\mathrm{So}=\mathrm{Sc}=0.2, R=7$; (d) $k p=0.5, M^{*}=2, \operatorname{Pr}=\mathrm{Ec}=\mathrm{Sc}=\mathrm{So}=s=0.2, R=7 ;$ (e) $k p=0.5, \operatorname{Pr}=\mathrm{Ec}=\mathrm{Sc}=\mathrm{So}=n=s=0.2, R=7 ;(\mathrm{f})$ $M=2, \mathrm{Pr}=\mathrm{Ec}=\mathrm{Sc}=\mathrm{So}=n=s=0.2, R=7 ;(\mathrm{g}) k p=0.5, M=2, \mathrm{Ec}=\mathrm{Sc}=\mathrm{So}=n=s=0.2, R=7 ;$ (h) $k p=0.5, M=2, \operatorname{Pr}=\mathrm{Sc}=\mathrm{So}=n=s=0.2$, $R=7 ; \quad$ (i) $\quad k p=0.5, \quad M=2, \quad \operatorname{Pr}=\mathrm{Ec}=\mathrm{So}=\mathrm{Sc}=n=s=0.2 ; \quad(\mathrm{j}) \quad k p=0.5, \quad M=2, \quad \operatorname{Pr}=\mathrm{Ec}=\mathrm{Sc}=n=s=0.2, \quad R=7 ; \quad(\mathrm{k}) \quad k p=0.5$, $\operatorname{Pr}=\mathrm{Ec}=\mathrm{Sc}=\mathrm{So}=n=s=0.2, R=7 ;$ (l) $M=2, \operatorname{Pr}=\mathrm{Ec}=\mathrm{Sc}=\mathrm{So}=n=s=0.2, R=7 ;(\mathrm{m}) k p=0.5, M=2, \operatorname{Pr}=\mathrm{Ec}=\mathrm{So}=n=s=0.2, R=7 ;(\mathrm{n})$ $k p=0.5, M=2, \operatorname{Pr}=\mathrm{Ec}=\mathrm{Sc}=n=s=0.2, R=7 ;(\mathrm{o}) k p=0.5, M=2, \operatorname{Pr}=\mathrm{Ec}=\mathrm{Sc}=n=s=0.2, R=7$.

with the increasing value of $\mathrm{Sr}$, the concentration profile is also showing an increasing behavior.

\section{Conclusion}

The steady flow of the viscous incompressible Newtonian fluid having a magnetic field with porous influence is analyzed under the viscous dissipation and ohmic dissipation effects by using similarity transformation and assisting BCs. The system of coupled nonlinear ODEs is tackled with the help of computational software (MAPLE) by using various standard techniques. These techniques are considered effective and convenient in the literature. The findings of this study are presented via tables and graphs, which are in good agreement by physical behavior already discussed in the literature. The following points are to be noticed in the whole discussion: (i) The velocity profile decreases with the increasing magnetic parameter $(M)$, while reverse effects are observed in the case of thermal and concentration profiles.

(ii) All the three profiles show an increasing behavior when the porosity parameter $(k p)$ increases.

(iii) Temperature and concentration profiles decrease with the value of $\operatorname{Pr}$ and Sc, respectively.

(iv) Temperature boundary layer thickness expands with the increasing value of Ec and $R$.

(v) The concentration profile gets larger with the increasing value of Sr.

\section{Data Availability}

The data used to support the findings of this study are included within the article. 


\section{Conflicts of Interest}

The authors declare that they have no conflicts of interest.

\section{References}

[1] R. Cortell, "Effect of viscous dissipation and radiation on the thermal boundary layer over a nonlinearly stretching sheet," Physics Letters A, vol. 372, no. 5, pp. 631-636, 2008.

[2] X.-H. Su and L.-C. Zheng, "Approximate solution to MHD Falkner-Skan flow over permeable wall," Applied Mathematics and Mechanics, vol. 32, no. 4, pp. 401-408, 2011.

[3] M. U. Ahammad and M. S. H. Mollah, "Numerical study of MHD free convection flow and mass transfer over a stretching sheet considering Dofour and Soret effects in the presence of magnetic field," IJET-IJENS, vol. 11, no. 5, pp. 4-11, 2011.

[4] J. Alinejad and S. Samarbakhsh, "Viscous flow over nonlinearly stretching sheet with effects of viscous dissipation," Journal of Applied Mathematics, vol. 2012, Article ID 587834, 10 pages, 2012.

[5] V. K. Verma and A. K. Gupta, "MHD flow in a porous channel with constant suction/injection at the walls," International Journal of Pure and Applied Mathematics, vol. 118, no. 1, pp. 111-123, 2018.

[6] O. Anwar Bég, M. M. Rashidi, M. T. Rastegari, T. A. Bég, S. S. Motsa, and A. Halim, "DTM-padé numerical simulation of electrohydrodynamic ion drag medical pumps with electrical hartmann and electrical reynolds number effects," Journal of Advanced Biotechnology and Bioengineering, vol. 1, no. 2, pp. 62-79, 2013.

[7] R. Bhargava and H. Chandra, "Numerical simulation of MHD boundary layer flow and heat transfer over a nonlinear stretching sheet in the porous medium with viscous dissipation using hybrid approach," Journal of Arxiv Fluid Dynamics (physics.Flu-Dyn), vol. 17, no. 1, pp. 1-19, 2017.

[8] R. A. Oderinu and Y. A. S. Aregbesola, "Analysis of skin friction in MHD Falker-Skan flow problem," Journal of the Nigerian Mathematical Society, vol. 34, no. 2, pp. 195-199, 2015.

[9] H. Dessie and N. Kishan, "MHD effects on heat transfer over stretching sheet embedded in porous medium with variable viscosity, viscous dissipation and heat source/sink," Ain Shams Engineering Journal, vol. 5, no. 3, pp. 967-977, 2014.

[10] P. S. Reddy and A. J. Chamkha, "Soret and dufour effects on unsteady MHD heat and mass transfer from a permeable stretching sheet with thermophoresis and non-uniform heat generation/absorption," Journal of Applied Fluid Mechanics, vol. 9, no. 5, pp. 2443-2455, 2016.

[11] R. Cortell, "MHD (magneto-hydrodynamic) flow and radiative nonlinear heat transfer of a viscoelastic fluid over a stretching sheet with heat generation/absorption," Energy, vol. 74, no. 1, pp. 896-905, 2014.

[12] R. P. Sharma, M. Jain, S. R. Munjam, and D. Kumar, "Effects of ohmic dissipation and chemical reaction on MHD free convection flow through porous medium with thermal radiation," Journal of Ultra Scientist of Physical Sciences Section A, vol. 29, no. 08, pp. 315-326, 2017.

[13] J. Pattnaik, C. Dash, and L. Ojha, "MHD falkner-skan flow through porous medium over permeable surface," Modelling, Measurement and Control B, vol. 86, no. 2, pp. 380-395, 2017.

[14] D. Prakash, P. Suriyakumar, N. Sivakumar, and B. Rushi Kumar, "Influence of viscous and ohmic heating on MHD flow of nanofluid over an inclined nonlinear stretching sheet embedded in a porous medium," International Journal of
Mechanical Engineering and Technology (IJMET), vol. 9, no. 8, pp. 992-1001, 2018.

[15] S. Jagadha, K. Kalyani, and N. Kishan, "Viscous and ohmic dissipation on non-darcy MHD nanofluid mixed convection flow in porous medium with suction/injection effects," Journal of Physics, vol. 1, no. 1, pp. 1742-6596, 2019.

[16] I. Ullah, M. T. Rahim, H. Khan, and M. Qayyum, "Analysis of various semi numerical schemes for magnetohydrodynamic (MHD) squeezing fluid flow in porous medium," Propulsion and Power Research, vol. 8, no. 1, pp. 69-78, 2019.

[17] K. Jabeen, M. Mushtaq, and R. M. Akram, "A comparative study of MHD flow analysis in a porous medium by using differential transformation method and variational iteration method," Journal of Contemporary Applied Mathematics, vol. 9, no. 2, pp. 1-15, 2019.

[18] F. Mabood and S. Shateyi, "Multiple slip effects on MHD unsteady flow heat and mass transfer impinging on permeable stretching sheet with radiation," Modelling and Simulation in Engineering, vol. 2019, Article ID 3052790, 11 pages, 2019.

[19] A. Emeka, "Effect of viscous dissipation and soret on MHD flow with thermal radiation," International Journal of Applied Science and Mathematical Theory, vol. 5, no. 1, pp. 57-65, 2019.

[20] F. Mabood and N. Pochai, "Analytical investigation of magnetohydrodynamic flow over a nonlinear porous stretching sheet," Advances in Mathematical Physics, vol. 2016, Article ID 7821405, 6 pages, 2016. 